\title{
O pH DAS SOLUÇÕES NUTRITIVAS NO COMPORTAMENTO DE CULTIVARES DE TRIGO À TOXICIDADE DE ALUMÍNIO (1)
}

\author{
CARLOS EDUARDO DE OLIVEIRA CAMARGO (2). Seçāo de Arroz e Cereais de Inverno. \\ Instituto Agronsmico.
}

\section{RESUMO}

Foram estudados nove cultivares de trigo em soluções nutritivas contendo quatro níveis de alumínio $(0,5,10$ e 20mg/litro) combinados com três níveis de $\mathrm{pH}(4,0,5,0$ e 6,0). A tolerância foi medida pela capacidade de as raizes primárias continuarem a crescer em solução sem alumínio, após um período de permanência de 48 horas em solução contendo determinados niveis cle $\mathrm{pH}$ e de alumínio. Os cultivares BH-1146, IAC-18, IAC-13 e C-3 foram tolerantes; IAC-17 e Alondra-4546 foram moderadamente tolerantes, e Siete Cerros, Super-x e CNT-8 foram sensiveis à presença de quantidades crescentes de $\mathrm{Al}^{3}+$ nas soluções de tratamentos quando foi mantido o pH 4,0. Todos os cultivares foram tolerantes às dosagens de alumínio estudadas quando foram mantidos os níveis de $\mathrm{pH}$ 5,0 ou 6,0 . Ficou confirmado que um controle rigoroso do $\mathrm{pH}$ da solução tratamento é um fator de grande importância no estudo da toxicidade do alumínio a diferentes cultivares de trigo.

\section{NNTRODUĞ̃̃O}

Muitos pesquisadores têm usado o solo para a separação de grande número de cultivares de trigo tolerantes ao alumínio $(\mathbf{3 , 8 , 1 1})$. A desvan-

(1) Com verba suplementar do Acordo do Trigo entre as Cooperativas de Produtores Rurais do Vale do Paranapanema e a Secretaria de Agricultura e Abastecimento, por meio do Instituto Agronômico. Recebido para publicação a 26 de março de 1982.

(2) Com bolsa de suplementação do CNPq. 
tagem de usar o solo é que o grau de seleção de plantas tolerantes não pode ser quantitativamente controlado, pois a solubilidade do alumínio e a severidade de sua toxicidade são afetadas por muitos fatores do solo (1). KERRIDGE ( $\boldsymbol{\gamma}$ ) descreveu uma técnica de separação de variedades de trigo tolerantes ao alumínio empregando soluções nutritivas nas quais eram mantidos rigorosamente o pH e a concentração aa solução, em uma câmara de crescimento com temperatura controlada. $\mathrm{O}$ mesmo autor mostrou diferenças quantitativas entre grupos de variedades de trigo.

Num estudo de dez cultivares de trigo em soluçōes nutritivas contendo três diferentes níveis de alumínio tóxico combinados com quatro diferentes concentrações salinas, foi demonstrado que o sintoma de toxicidade do aluminio, paralisação do crescimento radicular, ficou acentuado pelo aumento da concentração de alumínio na solução ou pela diminuição de concentração de sais para todos os cultivares estudados. Aparentemente, tolerância à toxicidade de determinada concentraçāo de alumínio é uma característica antes relativa do que absoluta, por depender da concentração de sais presentes (5).

Sintomas de toxicidade ao alumínio foram obtidos em soluções nutritivas para oito cultivares de trigo, tanto por um aumento da concentração de aluminio, mantendo constante a temperatura das soluçōes, quanto pelo aumento da temperatura, mantendo constante: determinada quantidade de aluminio (2).

De acordo com a teoria da hidrólise, há dez vezes mais alumínio na forma trivalente $\left(\mathrm{Al}^{3+}\right)$ do que na bivalente $\left(\mathrm{AlOH}^{2+}\right)$ em condições de $\mathrm{pH}$ igual a 4,0;3,1 vezes em $\mathrm{pH}$ igual a 4,5, enquanto em $\mathrm{pH}$ igual a 5,0 as quantidades de aluminio nas formas trivalentes e bivalentes sāo praticamente iguais. Diferentes espécies de plantas ou cultivares de uma mesma espécie em solução nutritiva podem mudar o $\mathrm{pH}$, devido a uma desigual absorção entre cátions e ânions $(\mathbf{6 , 9})$, causando um efeito significativo sobre a quantidade e a forma do alumínio presente na soluçāo. Com base nessas consideraçōes, pode ser facilmente entendido que um controle rigoroso do $\mathrm{pH}$ da solução é um fator importante no estudo da toxicidade do alumínio às plantas, porém há muito poucos trabalhos na literatura onde preciso controle do $\mathrm{pH}$ foi mantido (10).

$O$ presente trabalho tem por objetivo estudar o comportamento de cultivares de trigo a diferentes níveis de alumínio en soluções nutritivas, mantendo um $\mathrm{pH}$ constante, e o efeito de diferentes níveis de $\mathrm{pH}$ nas soluções nutritivas mantendo constante determinada concentração de alumínio.

\section{MATERIAL E MÉTODOS}

Os cultivares de trigo estudados foram os seguintes: IAC-17, BH-1146, CNT-8, IAC-13, IAC-18, Alondra-4546, C-3, Siete Cerros e Super-x. 
O delineamento estatístico empregado foi parcelas subsubdivididas com duas repetições, onde as parcelas foram compostas por quatro diferentes concentraçōes de alumínio $(0,5,10$ e $20 \mathrm{mg} /$ litro) nas soluções tratamentos, as subparcelas por três diferentes níveis de $\mathrm{pH}(4,0,5,0 \mathrm{e}$ $6,0)$ e as subsubparcelas pelos nove cultivares relacionados.

As sementes dos nove cultivares, cuidadosamente lavadas com uma solução de hipoclorito de sódio a $10 \%$, foram colocadas para germinar em caixas de Petri por 24 horas. Após esse tempo, as radículas estavam iniciando a emergência.

Foram escolhidas vinte sementes uniformes de cada cultivar e colocadas sobre o topo de doze telas de náilon. Cada uma das telas foi colocada em contato com a solução nutritiva completa existente em vasilha plástica de 8,30 litros de capacidade cada uma.

A concentração da solução nutritiva completa foi a seguinte: $\mathrm{Ca}\left(\mathrm{NO}_{3}\right)_{2} 4 \mathrm{mM} ; \mathrm{MgSO}_{4} 2 \mathrm{mM} ; \mathrm{KNO}_{3} 4 \mathrm{mM}$; $\left(\mathrm{NH}_{4}\right)_{2} \mathrm{SO}_{4} 0,435 \mathrm{mM} ; \mathrm{KH}_{2} \mathrm{PO}_{4}$ $0,5 \mathrm{mM} ; \mathrm{MnSO}_{4} 2 \mu \mathrm{M}$; CuSO ${ }_{4} 0,3 \mu \mathrm{M} ; \mathrm{ZnSO}_{4} 0,8 \mu \mathrm{M}$; NaCl $30 \mu \mathrm{M}$; Fe-CYDTA $10_{\mu} \mathrm{M} ; \mathrm{Na}_{2} \mathrm{MoO}_{4} \quad 0,1_{\mu} \mathrm{M}$ e $\mathrm{H}_{3} \mathrm{BO}_{3} 1_{\mu} \mathrm{M}$. O nivel da solução nas vasilhas plásticas tocou embaixo da tela de náilon, de maneira que as sementes foram mantidas úmidas e as radiculas emergentes tinham um pronto suprimento de nutrientes. $\mathrm{O}$ pH da solução foi previamente ajustado para 4,0 com $\mathrm{H}_{2} \mathrm{SO}_{4} 1 \mathrm{~N}$ e as vasilhas contendo as soluções foram colocadas em banho-maria com temperatura de $25 \pm 1^{\circ} \mathrm{C}$ dentro do laboratório.

As plantas desenvolveram-se nessas condições por 48 horas. Após esse período, cada plântula tinha três raizes primárias, uma mais longa medindo cerca de $4,5 \mathrm{~cm}$ e duas mais curtas, localizadas lateralmente à primeira.

Cada uma das doze telas de náilon com as vinte plântulas de cada cultivar foi transferida das soluções nutritivas completas para vasilhas plásticas de mesma capacidade, contendo as soluçóes tratamentos com a décima parte da concentração de sais da solução completa acrescidas de diferentes concentrações de alumínio em diferentes niveis de $\mathrm{pH}$ conforme a relação seguinte:

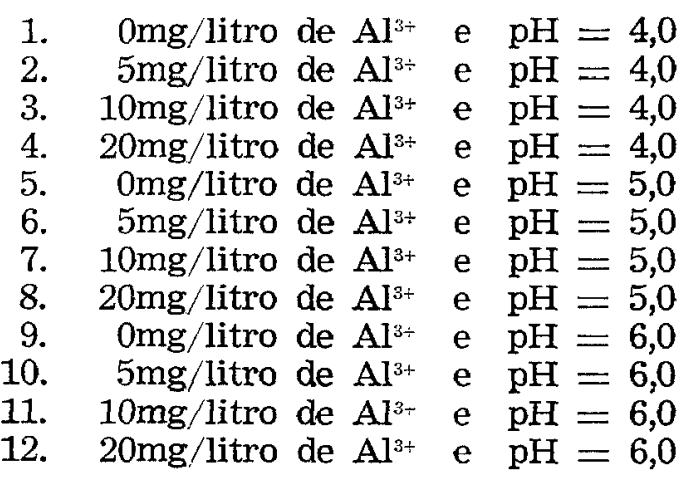


Nas soluçōes tratamentos, o fósforo foi omitido e o ferro adicionacio em quantidade equivalente àquela adicionada na solução completa como $\mathrm{FeCl}_{3}$ no lugar de Fe-CYDTA, como foi descrito por MOORE et alii (10). O fósforo foi omitido para evitar a possivel precipitação do alumínio. Antes da transferência das telas contendo as plântulas para as soluçōes de tratamento, foi adicionada a quantidade necessária de

$\mathrm{Al}_{2}\left(\mathrm{SO}_{4}\right)_{3} 18 \mathrm{H}_{2} \mathrm{O}$, de forma a serem obtidas soluçōes com $0,5,10$ e $20 \mathrm{mg}$ /litro de alumínio, e, em seguida, suficiente $\mathrm{H}_{2} \mathrm{SO}_{4} 1 \mathrm{~N}$ ou $\mathrm{NaOH} 1 \mathrm{~N}$,

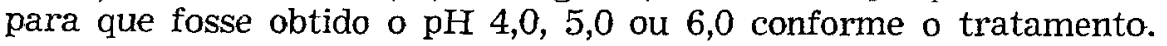

As plântulas ficaram crescendo por 48 horas nas respectivas soluções tratamentos em banho-maria com a temperatura de $25 \pm 1^{\circ} \mathrm{C}$. No final das 48 horas, a raiz primária de cada plântula foi medida, e, as plântulas, transferidas de volta para as doze vasilhas contendo as soluçōes nutritivas completas, onde cresceram antes das soluções tratamentos. Permaneceram crescendo nas soluçōes completas por mais 72 horas com temperatura de $25 \pm 1^{\circ} \mathrm{C}$. O crescimento das raízes nessas 72 horas depende da severidade do prévio tratamento. Com uma quantidade tóxica de alumínio, as raízes primárias não crescem mais e permanecem grossas, mostrando no ápice uma injúria típica, com descoloramento. A quantidade de crescimento da raiz foi determinada, medindo-se novamente o comprimento da raiz de cada plântula no final das 72 horas, na solução completa, e subtraindo o comprimento da mesma medida no final do crescimento nas soluções de tratamentos.

Durante todo o experimento o $\mathrm{pH}$ das soluções de tratamentos foi mantido o mais próximo possível de $4,0,5,0$ ou 6,0 , por ajustamentos diários com $\mathrm{H}_{2} \mathrm{SO}_{4}$ $1 \mathrm{~N}$ ou $\mathrm{NaOH} 1 \mathrm{~N}$. Foi empregada luz artificial e arejamento das soluções na totalidade do experimento.

Os dados foram analisados estatisticamente, considerando-se a média do crescimento da raiz das vinte plântulas de cada cultivar após a influência prejudicial do alumínio nas soluções de tratamento submetidas a diferentes niveis de $\mathrm{pH}$. A comparação entre as médias de crescimento da raiz dos nove cultivares dentro de uma mesma concentração de alumínio e um mesmo $\mathrm{pH}$ foi feita pelo teste de Tukey (in 12).

\section{RESULTADOS E DISCUSSÃo}

O comprimento médio das raízes dos nove cultivares de trigo, medido após 72 horas de crescimento na soluçáo nutritiva completa, seguido de 48 horas de crescimento nas soluções de tratamentos contendo os níveis de $\mathrm{pH} 4,0,5,0$ e 6,0 combinados com quatro diferentes concentrações de alumínio $(0,5,10$ e $20 \mathrm{mg} /$ litro), encontra-se no quadro 1.

Os resultados da análise estatística desse experimento são apresentados no quadro 2. Verificaram-se, pelo teste $F$, efeito significativo ao nível de $5 \%$ de concentrações de alumínio, e efeitos altamente significa- 


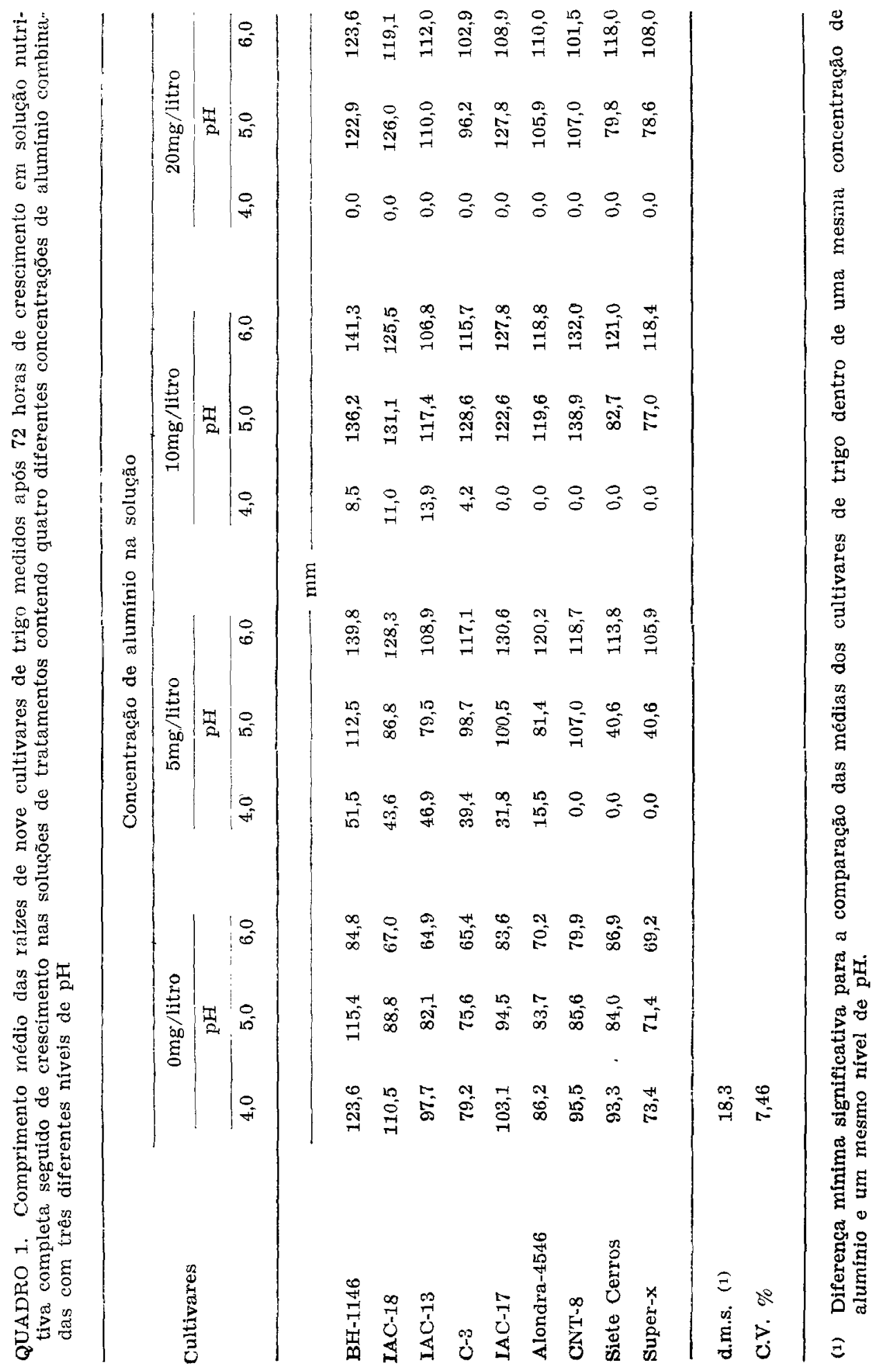


tivos de cultivares, níveis de $\mathrm{pH}$, níveis de $\mathrm{pH} \times$ concentrações de alumínio, cultivares $\mathrm{x}$ concentrações de alumínio, cultivares $\mathrm{x}$ niveis de $\mathrm{pH}$ e cultivares $\mathrm{x}$ concentrações de aluminio $\mathrm{x}$ niveis de $\mathrm{pH}$ nas soluções de tratamentos.

QLADRO 2. Análíse da variância para crescimento da raiz de nove cultivares de trigo após serem testados em soluções nutritivas contendo quatro diferentes concentrações de alumínio combinadas com três diferentes niveis de $\mathrm{pH}$

\begin{tabular}{|c|c|c|}
\hline Causas de variação & GLe & QM \\
\hline Repetiçőes & 1 & 11,03 \\
\hline Concentrações ãe $\mathrm{Al}^{3}$ & 3 & $1809,15 *$ \\
\hline Erro I & 3 & 73,74 \\
\hline $\mathrm{pH}$ & 2 & $124171,87 \% *$ \\
\hline $\mathrm{pH} \times$ concentraçōes de $\mathrm{Al}+4$ & 6 & $23688,86 * *$ \\
\hline Erro II & 8 & 144,01 \\
\hline Cultivares & 8 & $2516,37 *$ \\
\hline Cultivares $\mathrm{x}$ conc. de $\mathrm{Al}^{3+}$ & 24 & $289,03 * *$ \\
\hline Cultivares $\mathrm{x} \mathrm{pH}$ & 16 & $643,72 \%$ \\
\hline Cultivares $x$ conc. de $\mathrm{Al}^{3}+\mathrm{x}$ pH & 48 & $153,47 * *$ \\
\hline Erro III & 96 & 34,42 \\
\hline
\end{tabular}

Total

215

Considerando-se as médias dos diferentes cultivares estudados com 0mg/litro de aluminio nas soluçōes de tratamentos quando submetidos aos níveis de $\mathrm{pH}$ 4,0, 5,0 e 6,0, verificou-se como tendência geral uma diminuição do crescimento das raízes dos cultivares nas soluçōes nutritivas completas, com o aumento dos niveis de $\mathrm{pH}$.

Para a dosagem de $5 \mathrm{mg} /$ litro de $\mathrm{Al}^{3+}$ nas soluções de tratamentos, verificou-se que todos os cultivares foram tolerantes quando se empregaram os níveis de $\mathrm{pH} 5,0$ e 6,0. Os cultivares CNT-8, Siete Cerros e Super-x foram sensiveis a $5 \mathrm{mg} / \mathrm{litro}$ de $\mathrm{Al}^{3-}$ quando $\mathrm{o} \mathrm{pH}$ da solução foi 4,0 e os cultivares BH-1146, IAC-18, IAC-13, C-3, IAC-17 e Alondra-4546 foram tolerantes a essa condição.

Considerando-se a dosagem de 10 e $20 \mathrm{mg}$ /litro de $\mathrm{Al}^{3+}$, ficou demonstrado que, à medida que o pH da soluçáo tratamento aumentou de 4,0 para 6,0 , houve um aumento no crescimento das raízes de todos os cultivares estudados. 
Os cultivares Alondra-4546 e IAC-17 foram sensiveis quando se empregou uma solução tratamento com $\mathrm{pH} 4,0$ e contendo $10 \mathrm{mg} /$ litro de $\mathrm{Al}^{3+}$. Os cultivares $\mathrm{BH}-1146$, IAC-18, IAC-13 e C-3 foram tolerantes nessa condição.

Todos os cultivares foram sensiveis quando se adicionaram $20 \mathrm{mg} / \mathrm{li}$ tro de $\mathrm{Al}^{3+}$ na solução tratamento, mantendo-se constante $\mathrm{opH} 4,0$.

Com base nos resultados obtidos, verificou-se que, apesar do efeito marcante da elevação do $\mathrm{pH}$ na tolerância de um particular cultivar de trigo a uma mesma concentração de alumínio, três grupos de cultivares poderiam ser considerados: $\mathrm{BH}-1146$, IAC-18, IAC-13 e C-3 como os cultivares mais tolerantes; IAC-17 e Alondra-4546 como moderadamente tolerantes, e Siete Cerros, Super-x e CNT-8 como sensiveis.

Os resultados obtidos estão de acordo com aqueles concluídos por ALI (1), onde a influência prejudicial do alumínio foi dependente do $\mathrm{pH}$ das soluções tratamentos e a toxicidade de certa concentração de alumínio diminuiu com a elevação do $\mathrm{pH}$ de 4,0 para 7,0 , diminuindo, conseqüentemente, a solubilidade desse elemento.

Como foi concluido por CAMARGO (2), CAMARGO \& OLIVEIRA (4) e CAMARGO et alii (5) que a identificação de plantas de trigo tolerantes e sensiveis ao alumínio em soluções nutritivas é uma função da concentração de sais, da temperatura e da concentração de alumínio, foi mostrado no presente trabalho o efeito do $\mathrm{pH}$. Verificou-se a necessidade de que sejam controladas todas essas variáveis para que os resultados obtidos num experimento possam ser repetidos em outras oportunidades com a mesma precisão, evitando, pois, erros na identificação de plantas sensiveis e tolerantes, principalmente quando provenientes de populações híbridas segregantes, onde em geral são eliminadas as plantas sensiveis.

\section{CONCLUSÕES}

a) A identificação de cultivares de trigo tolerantes e sensiveis a determinadas concentrações de alumínio foi significativamente dependente do $\mathrm{pH}$ da solução nutritiva.

b) Houve um significativo aumento do comprimento das raizes dos cultivares estudados, mantendo-se constante determinada dosagem de $\mathrm{Al}^{3+}(5,10$ ou $20 \mathrm{mg}$ /litro) quando $\mathrm{o} \mathrm{pH}$ da solução variou de 4,0 para 6,0 , evidenciando que um controle rigoroso do $\mathrm{pH}$ da solução seria um fator de grande relevância no estudo da toxicidade do aluminio.

c) Os cultivares BH-1146, IAC-18, IAC-13 e C-3 foram os mais tolerantes; IAC-17 e Alondra-4546 foram moderadamente tolerantes, $\mathrm{e}$ Siete Cerros, Super-x e CNT-8 foram sensiveis entre os cultivares estudados a niveis crescentes de $\mathrm{Al}^{3+}$ nas soluções nutritivas de tratamentos, mantendo-se constante o pH 4,0. Todos os cultivares se apresentaram como tolerantes a todas as concentrações de aluminio estudadas quandō foram mantidos constantes os níveis de $\mathrm{pH} 5,0$ ou 6,0 . 


\section{SUMMARY}

\section{EFFECT OF pH IN NUTRIENT SOLUTION ON TOLERANCE TO ALUMINUM TOXICITY IN WHEAT CULTIVARS}

The aluminum tolerance of nine wheat cultivars was studied in nutrient solutions using three different levels of $\mathrm{pH}$ combined with four different concentrations of this element. The tolerance was evaluated by measuring the root growth in an aluminum-free complete nutrient solution after a previous treatment in aluminum added solutions $(0,5,10$ and $20 \mathrm{mg} / 1)$ under a particular $\mathrm{pH}(4.0,5.0$ and 6.0). The wheat cultivars $\mathrm{BH}-1146$, IAC-18, IAC-13 and C-3 presented tolerance, IAC-17 and Alondra-4546 showed moderate tolerance and Siete Cerros, Super- $\mathrm{x}$ and CNT-8 were sensitive to the presence of increasing concentrations of $\mathrm{Al}^{3+}$ in the treatment solution under $\mathrm{pH}$ 4.0. All cultivars were tolerant to the different concentrations of aluminum under $\mathrm{pH} 5.0$ and 6.0. The aluminum toxicity symptom (inhibition of root growth) was dependent on the $\mathrm{pH}$ and the amount of aluminum in the treatment solution. For the same level of aluminum, toxicity symptoms increased, when the $\mathrm{pH}$ decreased in the solution from 6.0 to 4.0 , considering all the studied cultivars, Apparently wheat tolerance to $\mathrm{Al}^{3+}$ is a relative rather than absolute characteristic, depending on $\mathrm{pH}$ among other characteristics.

\section{REFERÊNCLAS BLBLIOGRẢFICAS}

1. ALI, S. M. E. Influence of cations on aluminum toxicity in wheat (Triticum aestivum Vill, Host). Corvallis, Universidade Estadual de Oregon, 1973. 102p. Tese de Doutoramento.

2. CAMARgo, C. E. de O. Efeito da temperatura em solução nutritiva na tolerância ao alumínio de cultivares de trigo. Bragantia, Campinas, 42:51-63, 1983.

3. - ; ARMIGER, W. H.; BRIGGLE, L. W.; REID, D. A. Differential aluminum tolerance of wheat and barley varieties in acid soils. Agronomy Journal, 57:413-417, 1965.

4. —_ \& OLTVEIRA, O. F. Tolerância de cultivares de trigo a difercntes niveis de alumínio em solução nutritiva e no solo. Bragantia, Campinas, 40:21-31, 1981.

5. -__ _ _ _ LAVORENTI. A. Efeito de diferentes concentrações de sais em solução nutritiva na tolerância de cultivares de trigo à toxidez de alumínio. Bragantia, Campinas, 40:93-101, 1981.

6. FOY, C. D. \& FLEMTNG, A. L. The physiology of plant tolerance to excess available aluminum and manganese in acid soils. In: AMERICAN SOCIETY OF AGRONOMY. II. Crop tolerance to suboptimal land conditions: proceedings of a symposium held in Houston, Texas, edited by Gerald A. Jung. Madison, Wisconsin, 1978. p.301-328, (ASA Special Publications, 32)

7. KERRIDGE, P. C. Aluminum toxicity in wheat (Triticum aestivum Vill, Host). Corvallis, Universidade Estadual de Oregon, 1969. 170p. Tese de Doutoramento.

8. MESDAG, J.; SLOOTMAKER, L. A.; POST JR., J. Linkage between tolerance to high soil acidity and genetically high protein content in the kernel of wheat, Triticum aestivum L., and its possible use in breeding. Euphytica, $19: 163-174,1976$.

9. MOORE, D. P. Physiological effects of pH on plant roots. In: CARSON, E. W., ed. The plant root and its environment. Charlottesville, VA, Univ. Press cf Virginia, 1974. p.135-151. 
10. MOORE, D. P.; KRONSTAD, W. E.; METZGER R. Screening wheat for aluminum tolerance. In: WRIGHT, Madison J., ed. Workshop on Plant Adaptation to Mineral Stress in Problem Soils, Beltsville, Maryland, 1976. Proceedings. Ithaca, Cornell University, 1976. p.287-295.

11. REID, D. A. Genetics potentials for solving problems for soil mineral stress: aluminum and manganese toxicities in the cereal grains. In: WRIGHT, Madison J., ed. Workshop on Plant Adaptation to Mineral Stress in Problem Soils, Beltsville, Maryland, 1976. Proceedings. Ithaca, Cornell University, 1976. p.55-64.

12. STEEL, R. G. D. \& TORRIE, J. H. Principles and procedures of statistics. New York, Mc Graw-Hill, 1960. 481p. 